OPEN ACCESS

Edited by:

Alan Apter,

Schneider Children's Medical

Center, Israel

Reviewed by:

Marie-Laure Paillere Martinot,

Hôpitaux Universitaires Pitie

Salpêtrière, France

Martine F. Flament,

University of Ottawa, Canada

${ }^{*}$ Correspondence:

Ru Zhang

ru.zhang@boystown.org

Specialty section

This article was submitted to Child and Adolescent Psychiatry, a section of the journal

Frontiers in Psychiatry

Received: 13 October 2020 Accepted: 25 October 2021 Published: 16 November 2021

Citation:

Zhang R, Bashford-Largo J, Lukoff J,

Elowsky J, Carollo E, Schwartz A

Dobbertin M, Bajaj S, Blair KS,

Leibenluft $E$ and Blair RJR (2021)

Callous-Unemotional Traits Moderate

the Relationship Between Irritability

and Threatening Responding

Front. Psychiatry 12:617052.

doi: 10.3389/fpsyt.2021.617052

\section{Callous-Unemotional Traits Moderate the Relationship Between Irritability and Threatening Responding}

\author{
Ru Zhang ${ }^{1 *}$, Johannah Bashford-Largo ${ }^{1}$, Jennie Lukoff ${ }^{1}$, Jaimie Elowsky ${ }^{1}$, Erin Carollo ${ }^{1}$, \\ Amanda Schwartz ${ }^{1}$, Matthew Dobbertin ${ }^{1}$, Sahil Bajaj ${ }^{1}$, Karina S. Blair ${ }^{1}$, Ellen Leibenluft ${ }^{2}$ \\ and R. James R. Blair ${ }^{1}$ \\ ${ }^{1}$ Center for Neurobehavioral Research, Boys Town National Research Hospital, Boys Town, NE, United States, ${ }^{2}$ Section on \\ Mood Dysregulation and Neuroscience, Intramural Research Program, National Institute of Mental Health, National Institutes \\ of Health, Bethesda, MD, United States
}

Background: Irritability and callous-unemotional (CU; reduced guilt/empathy) traits vary dimensionally in the typically developing population but may be particularly marked in youth with conduct disorder (CD). While these dimensional traits are positively correlated, they have been associated with divergent forms of dysfunction, particularly with respect to threat processing (i.e., irritability with increased, and CU traits with decreased, threat responsiveness). This suggests that interactions between these two dimensions may be complex at the neurobiological level. However, this issue has received minimal empirical attention.

Methods: The study included 105 adolescents (typically developing and cases with CD; $N=59$ ). They were scanned with $\mathrm{fMRI}$ during a looming threat task that involved images of threatening and neutral human faces or animals that appeared to be either looming or receding.

Results: Significant irritability-by-CU traits-by-Direction-by-Emotion interactions were seen within right thalamus/PAG, left lingual gyrus and right fusiform gyrus; irritability was positively associated with the BOLD response for Looming Threatening vs. Receding Threatening trials, particularly for youth with low CU traits. In contrast, CU traits were negatively associated with the same differential BOLD response but particularly for youth showing higher levels of irritability. Similar findings were seen within left ventral anterior and posterior cingulate cortices, though the addition of the interaction with CU traits was only seen at slightly more lenient thresholds.

Conclusions: The results support previous work linking irritability to increased, and CU traits to reduced, threat responsiveness. However, for adolescents with high irritability, if $\mathrm{CU}$ traits are also high, the underlying neuropathology appears to relate to reduced, rather than increased, threat responsiveness.

Keywords: threat responsiveness, irritability, callous-unemotional traits, conduct disorder, fMRI 


\section{INTRODUCTION}

According to the fifth edition of Diagnostic and Statistical Manual of Mental Disorders [DSM-5; (1)], the essential feature of conduct disorder (CD) is a repetitive and persistent pattern of behavior in which the basic rights of others, or major ageappropriate social norms, are violated. Youth with conduct problems are at risk of emotional impairment and low social and academic achievement that can have long-term detrimental effects on developmental outcomes $(2,3)$. Adolescents showing conduct problems are a heterogeneous population who differ in age of onset, form of aggression (reactive or instrumental), comorbidity of affective disorders, treatment response and affective traits (2). Numerous efforts have been made to understand this heterogeneity in an attempt to tailor treatments and improve outcomes. An approach that has gained empirical support in recent years is to focus on affective traits related to CD and their neurobiology.

One area of neurobiological function that received focus is threat processing. Core brain regions implicated in threat processing include those generating the emotional response [amygdala, hypothalamus, periaqueductal gray (PAG), and anterior insula cortex]; those representing the threat (visual and temporal cortices); and those representing emotional valence and maintaining the emotional reaction [ventromedial prefrontal cortex (vmPFC), rostromedial prefrontal cortex (rmPFC), and posterior cingulate cortex (PCC)] (4-9).

Affective traits related to conduct problems include: (i) callous-unemotional (CU) traits (reduced guilt following misbehavior, reduced empathy for others in distress, reduced concern about one's own performance, and shallow or deficient affect) (10); and (ii) irritability-defined as an "increased propensity to exhibit anger relative to one's peers" [(11), p.277] and a "relative dispositional tendency to respond with anger to blocked goal attainment, and includes both mood (trait) and behavioral (reactive state) dysregulation" $[(12,13)$, p.69; see also $(14,15)]$. Work on CU traits and irritability has, for the most part, developed in parallel [though see $(4,15)]$. Most studies indicate that the level of CU traits is inversely related to responsiveness to the distress of others and to threat stimuli in the amygdala and related regions such as anterior insula, temporal cortices and vmPFC (16-18). In contrast, neuroscience-based models of irritability typically stress hyperactivity to threat $(4,11,19)$. These findings of threat hypo-responsiveness relating to $\mathrm{CU}$ traits but threat hyper-responsiveness relating to irritability are striking given that $\mathrm{CU}$ traits and irritability are positively associated [e.g., $r=0.463$; (20)]. Indeed, irritability may predict later CU levels (21) - though it has also been associated with later depression (22).

The question then becomes how to understand the relationship between the neurobiology and presentation of $\mathrm{CU}$ traits vs. irritability. Is irritability underpinned by the same neurobiology whatever the individual's level of CU traits? Or could irritability be underpinned by different neurobiology in individuals with high vs. low CU traits? Given that: (i) higher irritability has been associated with greater threat responsiveness (23-27); but (ii) higher CU traits has been associated with lower threat responsiveness (16-18); and (iii) irritability and CU traits are positively associated, it suggests that the neuro-cognitive models of irritability and/or CU traits may be incomplete for adolescents with high levels of irritability and CU traits. This should manifest as irritability-by-CU traits interactions with respect to BOLD response data such that the relationship between irritability (and/or CU) traits should differ as a function of level of CU traits (and/or irritability).

The current study explores these issues. Specifically, the current study examines the extent to which there is an interaction between level of $\mathrm{CU}$ traits and irritability in adolescents, specifically in neural systems responsive to threat. Consistent with $\mathrm{RDoC}(28,29)$, a dimensional approach was taken, with the sample representing the range from typically developing (TD) to a psychiatrically heterogeneous (in terms of comorbid diagnoses) sample of cases with $\mathrm{CD}$ (a psychiatric condition particularly associated with CU traits and irritability). We predicted that regions responsive to threat (e.g., the amygdala, PAG and connected cortical regions; vmPFC and visual and temporal cortices) would show irritability-by-CU traits interactions particularly manifesting at higher levels of irritability and/or CU traits. This prediction was assessed via two different analysis approaches. First, a standard task-by-CU traits/irritability ANCOVA analysis of the BOLD responses of the study participants. Second, an interrogation of BOLD response activity within regions implicated in task features (looming threat, threat images), via an independent TD sample, in the study participants (anonymous reviewer's suggestion).

\section{METHODS}

\section{Participants}

Study participants included 105 youths aged 14-18 years from a residential care program and the surrounding community (54 from the residential treatment program and 51 from the community): average age $=16.299(\mathrm{SD}=1.248)$, average $\mathrm{IQ}=99.829(\mathrm{SD}=9.689)$, and 63 males. An additional three participants from the residential treatment program were approached but either their parents did not provide consent or they did not assent. Participants were recruited as part of a broader study determining neural correlates of behavioral and emotional problems in youth. Participants were clinically characterized through psychiatric interviews conducted by a licensed, board-certified psychiatrist with the participants and their parents, to adhere closely to common clinical practice. A member of the clinical research team obtained written informed consent and assent. In all cases, youth had the right to decline participation at any time before or during the study. The Boys Town National Research Hospital institutional review board approved this study. For full details on consenting and assenting procedure and the exclusion criterion, see Supplementary Material.

\section{Measures fMRI Task}

The participants performed a looming task [adapted from (30)] and used previously with adolescent participants [e.g., (31-34)]. 


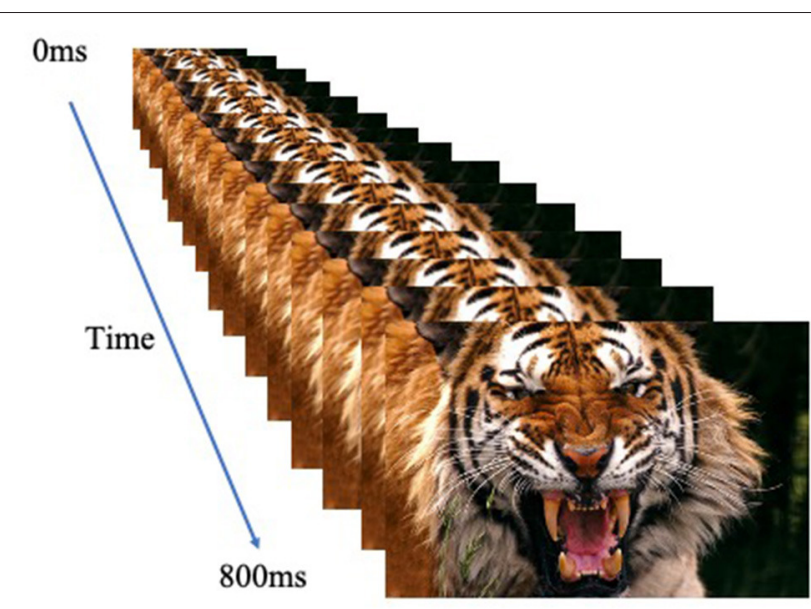

FIGURE 1 | The looming task. Example of the Looming Threatening Animal trial.

They were presented with an image that appeared to either loom toward or recede away from them. Images were human or animal faces and were either threatening or neutral. Images were presented rapidly in a series of sixteen $50 \mathrm{~ms}$ frames of increasing or decreasing size in the center of the screen to create the effect of either looming (i.e., increasing in size in rapid succession) or receding (i.e., decreasing in size in rapid succession; total stimulus duration: $800 \mathrm{~ms}$ ); see Figure 1. Stimulus presentations were followed by a fixation point, which was on screen for a jittered duration of 1,250-4,250 ms. The task included 1 block of 160 stimuli (20 of each of the 8 trial types). In order to ensure attention to the task, participants were instructed to press a button with their right index finger as quickly as possible when an image appeared on the screen.

\section{Inventory of Callous-Unemotional Traits (ICU)}

The ICU is a 24 -item self-report scale designed to assess CU traits in youth (35). The construct validity of the ICU has been supported in community and juvenile justice samples $(36,37)$.

\section{Affective Reactivity Index (ARI)}

It is a seven-item self-report questionnaire that assesses the youth's irritability during the preceding 6 months (six symptom items and one function impairment item). Prior work has indicated that the ARI is a reliable and valid measure of irritability in youth (14).

Additional measures included the conduct problems subscale from the Strength and Difficulties Questionnaire [SDQ-CP: (38)] and the Reactive Proactive Questionnaire (39) - to provide information on severity of aggression and association of aggression with $\mathrm{CU}$ traits and irritability in this population, the Conners ADHD scale (40), to provide information on severity of ADHD symptomatology in this population and the Alcohol Use Disorder Identification Test [AUDIT; (41)] and Cannabis Use Disorder Identification Test [CUDIT; (42)] assessing AUD and CUD symptom levels over the previous 12 months.

\section{fMRI Parameters}

Whole-brain BOLD fMRI data were acquired using a 3.0 Tesla Siemens Skyra scanner. Functional images were taken with a T2* weighted gradient echo planar imaging (EPI) sequence (repetition time $=2,500 \mathrm{~ms}$; echo time $=27 \mathrm{~ms} ; 240 \mathrm{~mm}$ field of view; $94 \times 94$ matrix; $90^{\circ}$ flip angle). Whole-brain coverage was obtained with 43 axial slices (thickness, $2.5 \mathrm{~mm}$; voxel size 2.6 $\times 2.6 \times 2.5 \mathrm{~mm}^{3}$ ). A high-resolution T1 anatomical scan (MPRAGE, repetition time $=2,200 \mathrm{~ms}$; echo time $=2.48 \mathrm{~ms} ; 230 \mathrm{~mm}$ field of view; $8^{\circ}$ flip angle; $256 \times 208$ matrix; thickness, $1 \mathrm{~mm}$; voxel size $0.9 \times 0.9 \times 1 \mathrm{~mm}^{3}$ ) in register with the EPI data set was obtained covering the whole brain with 176 axial slices.

\section{fMRI Analyses}

Data were analyzed within the framework of a random effects general linear model (GLM) using Analysis of Functional Neuroimages [AFNI; (43)]. The anatomical scan for each participant was registered to the Talairach and Tournoux atlas (44) and each participant's functional EPI data were registered to their Talairach anatomical scan in AFNI. Functional images were motion corrected to a reference volume and spatially smoothed with a 6-mm full width half maximum Gaussian kernel. The data then underwent time series normalization to a T1 image, and these results were multiplied by 100 for each voxel. Therefore, the resultant regression coefficients are representative of a percentage of signal change from the mean.

Afterward, eight indicator regressors were generated: Receding Neutral Animal, Receding Neutral Human, Receding Threatening Animal, Receding Threatening Human, Looming Neutral Animal, Looming Neutral Human, Looming Threatening Animal, Looming Threatening Human. General Linear Model fitting was performed with the eight regressors listed, six motion regressors, and a regressor modeling a firstorder baseline drift function. This produced a $\beta$-coefficient and an associated $t$-statistic for each voxel and regressor.

Volumes were censored if there was $>0.5 \mathrm{~mm}$ motion across adjacent volumes. Participants were excluded due to excessive motion ( $>20 \%$ censored volumes; mean $=0.7 \%, \mathrm{SD}=1.7 \%$ ) or low response rate $(<65 \%$ responses; mean $=91.6 \%$, SD $=6.5 \%)$ on the task. There were no significant correlations between ARI scores and censored volumes, average motion per volume, and maximum displacement during scanning within the final sample ( $r$ 's $=0$ to $0.08, p>0.40$ ). However, there was a negative correlation between ICU scores and average motion per volume $(r=-0.23, p=0.023)$. The correlations between ICU and censored volumes and maximum displacement were non-significant $(r=-0.18,-0.01, p=0.082,0.920)$.

\section{Statistical Analyses \\ Clinical Data}

Potential relationships between ICU and ARI scores and age, IQ, sex and prescribed medications were examined via zero-order correlation analyses.

\section{Behavioral Data}

To examine associations between ICU and ARI scores and behavioral performance on the task, we ran a full factorial 2 
(Direction: Looming, Receding) by 2 (Type: Animal, Human) by 2 (Emotion: Threatening, Neutral) repeated measures analysis of covariance (ANCOVA) on the response time (RT) data with ICU and ARI scores as continuous covariates. To reduce skewness and kurtosis (and thus disproportionate influence on coefficient estimates of data points in the tails/outliers), a rankit transformation (45) was applied to the ICU and ARI scores. Pre-transformation, these were 1.43 and 1.56 for the ARI and 0.47 and 0.11 for the ICU, respectively. Following transformation these were 0.02 and -0.20 and 0.49 and -0.36 , respectively. The rankit transformed ICU and ARI scores were then $z$-scored, and these values were used as continuous covariates in all ANCOVA analyses.

\section{BOLD Response Data}

(1) Whole brain analysis

To examine associations between ICU and ARI scores and dysfunction within brain regions responsive to Direction, stimulus Type or Emotion, we ran a full factorial 2 (Direction: Looming, Receding) by 2 (Type: Animal, Human) by 2 (Emotion: Threatening, Neutral) repeated measures ANCOVA on the BOLD response data with ICU and ARI scores as continuous covariates via $3 \mathrm{dMVM}$ within AFNI; i.e., all component main effects, and all two- and threeway interactions were included in the model. Group was not included as a variable in the statistical model as including $\mathrm{CD}$ as a covariate in the model would effectively divide the statistical variance between behavioral manifestations (CD behaviors and the socio-affective traits) of this diagnosis. Age, sex and IQ were not included in these models as none of these variables were associated with either ICU or ARI score.

(2) Functional ROI analysis

Functional ROIs were derived from the data from an independent sample of 99 TD participants (for details of the sample, see Supplementary Material 3). Specifically, to identify regions involved in threat processing, we conducted a full factorial 2 (Direction: Looming, Receding) by 2 (Type: Animal, Human) by 2 (Emotion: Threatening, Neutral) repeated measures ANOVA on the BOLD response data from this independent sample to identify regions showing a main effect of Direction (Looming > Receding) and/or Emotion (Threatening $>$ Neutral).

BOLD responses within the functional ROIs showing a main effect of Direction (Looming > Receding) and/or Emotion (Threatening $>$ Neutral) to Direction and/or Emotion cues for the study sample was then calculated. Following this, two Region by Direction by Type by Emotion repeated measures ANCOVAs were conducted on these data with ICU and ARI scores as continuous covariates (one for the Direction and one for the Emotion ROIs).

\section{Secondary Analyses}

Analyses exploring the nature of observed ANOVA and ANCOVA derived interactions were performed within RStudio and freely available online tools. In addition, Steiger's $z$-tests were used to compare the correlations between ICU and ARI scores and RT and BOLD responses to task variables (46).

For irritability-by-CU traits interactions, a bootstrapping procedure $(10,000$ samples $)$ using the PROCESS macro for SPSS (47) was used to examine how irritability/CU traits moderated the association of trait variables with RT or BOLD response. The Johnson-Neyman technique was used to investigate heterogeneity of the relationships between $\mathrm{CU}$ traits and irritability and BOLD responses (48). This technique identified specific ranges of trait scores (e.g., ARI scores) where the relationship between CU level (ICU scores) and RT/BOLD responses was significant (48).

\section{Secondary Analyses: CD Diagnosis and Potential Confounds CD Diagnosis}

To determine the relationship between $\mathrm{CD}$ diagnostic status and BOLD response, a full factorial 2 (Group: with CD, TD) by 2 (Direction: Looming, Receding) by 2 (Type: Animal, Human) by 2 (Emotion: Threatening, Neutral) repeated measures ANOVA was conducted on the BOLD response data.

\section{Potential Treatment Confounds}

Given potential associations of ICU and ARI scores with medication, our ANCOVA analysis was repeated twice, once excluding participants prescribed SSRIs $(N=10)$ and once excluding participants prescribed antipsychotics $(N=8)$.

\section{For All BOLD Response Analyses}

Correction for multiple comparisons was performed using a spatial clustering operation in AFNI's 3dClustSim utilizing the autocorrelation function (-acf) with 10,000 Monte Carlo simulations for the whole-brain analysis. Spatial autocorrelation was estimated from residuals from the individual-level GLMs. The initial threshold was set at $p=0.001$. This process yielded an extant threshold of $k=23$ voxels for the whole brain (multiple comparison corrected $p<0.05$ ).

\section{RESULTS}

\section{Clinical Data}

ICU and ARI were positively correlated; see Table 1. While neither were related to age, sex or IQ, both were positively correlated with prescription of antipsychotic and/or SSRI medications (see Table 1). They were also correlated with CUDIT and AUDIT scores-though the strength of correlations (ICU vs. ARI) did not significantly differ (see Table 1).

\section{fMRI Data}

\section{Whole Brain Analysis}

The covariate-based analysis revealed regions showing the following two interactions (also see Table 2): irritability-by-CU traits-by-Direction-by-Emotion interaction and irritability-byDirection-by-Emotion interaction. Regions showing main effects 
TABLE 1 | Demographic and clinical variables.

\begin{tabular}{|c|c|c|c|c|c|c|c|c|c|c|c|}
\hline & Mean for CD & Mean for TD & $\begin{array}{l}t \text { score for } \\
\text { TD vs. CD }\end{array}$ & $P$-value & $\begin{array}{l}\text { Mean for the } \\
\text { whole sample }\end{array}$ & Std. deviation & Range & Correlation with ARI & Correlation with ICU & Steiger's Z & $P$-value \\
\hline Age & 16.393 & 16.177 & -0.881 & 0.381 & 16.299 & 1.248 & $14.07-18.88$ & 0.033 & 0.085 & -0.513 & 0.608 \\
\hline$I Q$ & 98.746 & 101.217 & 1.301 & 0.196 & 99.829 & 9.689 & $79-123$ & 0.074 & -0.061 & 1.332 & 0.183 \\
\hline ARI & 3.627 & 0.913 & -5.798 & 0.000 & 2.438 & 2.728 & $0-11$ & - & $0.473^{\star \star}$ & - & - \\
\hline ICU & 26.246 & 15.767 & -6.459 & 0.000 & 21.740 & 9.542 & $3-51$ & $0.473^{\star \star}$ & - & - & - \\
\hline SDQ-CP & 7.102 & 0.087 & -27.838 & 0.000 & 4.029 & 3.722 & $0-10$ & $0.474^{\star \star}$ & $0.486^{\star \star}$ & -0.140 & 0.888 \\
\hline RPRS Total & 18.614 & 8.205 & -10.901 & 0.000 & 13.723 & 6.778 & $6-30$ & $0.446^{\star \star}$ & $0.453^{\star \star}$ & -0.080 & 0.936 \\
\hline RPRS Reactive & 11.114 & 4.872 & -10.691 & 0.000 & 8.181 & 4.097 & $3-15$ & $0.401^{\star \star}$ & $0.410^{\star \star}$ & -0.099 & 0.921 \\
\hline RPRS Proactive & 7.500 & 3.333 & -8.892 & 0.000 & 5.542 & 2.977 & 3-15 & $0.464^{* *}$ & $0.466^{\star *}$ & -0.023 & 0.982 \\
\hline Conners (ADHD) & 9.915 & 0.196 & -10.306 & 0.000 & 5.657 & 6.800 & $0-20$ & $0.531^{\star \star}$ & $0.371^{\star \star}$ & 1.842 & 0.066 \\
\hline AUDIT & 4.89 & 0.37 & -4.306 & 0.000 & 2.68 & 5.392 & $0-34$ & 0.182 & $0.274^{*}$ & -0.841 & 0.400 \\
\hline \multirow[t]{2}{*}{ CUDIT } & 10.96 & 0.49 & -7.105 & 0.000 & 5.84 & 8.653 & $0-29$ & $0.366^{* *}$ & $0.479^{* *}$ & -1.137 & 0.256 \\
\hline & Percent for CD & Percent for TD & & & $\mathbf{N}$ & Percent & & & & & \\
\hline Male & $62.7 \%$ & $56.5 \%$ & -0.638 & 0.525 & 63 & $60 \%$ & - & -0.119 & 0.018 & -1.039 & 0.299 \\
\hline$C D$ & $100 \%$ & $0 \%$ & - & - & 59 & $56.2 \%$ & - & $0.496^{\star \star}$ & $0.546^{\star \star}$ & -0.450 & 0.653 \\
\hline ADHD & $77.9 \%$ & $0 \%$ & -12.636 & 0.000 & 46 & $43.8 \%$ & - & $0.508^{\star \star}$ & $0.423^{\star \star}$ & 0.639 & 0.523 \\
\hline MDD & $18.6 \%$ & $0 \%$ & -3.216 & 0.002 & 11 & $10.5 \%$ & - & $0.495^{\star \star}$ & $0.481^{\star \star}$ & 0.046 & 0.963 \\
\hline GAD & $35.6 \%$ & $0 \%$ & -4.994 & 0.000 & 21 & $20 \%$ & - & $0.349^{\star \star}$ & $0.405^{\star \star}$ & -0.256 & 0.798 \\
\hline No diagnosis & $0 \%$ & $100 \%$ & - & - & 46 & $43.8 \%$ & - & $-0.496^{\star *}$ & $-0.546^{\star *}$ & 0.450 & 0.653 \\
\hline Antipsychotic & $13.6 \%$ & $0 \%$ & -2.660 & 0.009 & 8 & $7.6 \%$ & - & $0.231^{\star \star}$ & $0.268^{\star \star}$ & -0.084 & 0.933 \\
\hline Stimulant & $23.7 \%$ & $0 \%$ & -3.747 & 0.000 & 14 & $13.3 \%$ & - & 0.143 & 0.045 & 0.319 & 0.749 \\
\hline SSRI & $16.9 \%$ & $0 \%$ & -3.035 & 0.003 & 10 & $9.5 \%$ & - & 0.091 & $0.222^{\star}$ & -0.344 & 0.731 \\
\hline
\end{tabular}

IQ, Intelligent Quotient; ARI, Affective Reactivity Index; ICU, Inventory of Callous-Unemotional Traits; SDQ-CP, Strengths and Difficulties Questionnaire-Conduct Problem; RPRS, Reactive/Proactive Rating Scale; AUDIT, Alcohol Use Disorders Identification Test; CUDIT, Cannabis Use Disorder Test; CD, Conduct Disorder; ADHD, Attention Deficit Hyperactivity Disorder; MDD, Major Depressive Disorder; GAD, Generalized Anxiety Disorder; $P$ = two-tailed significance level of the Steiger's $Z$ calculation (i.e., whether there were significant differences in correlation strength between the ARI scores and ICU scores). ${ }^{*} P<0.05 .{ }^{* *} P<0.01$. 
TABLE 2 | Brain regions displaying significant task variable interactions with ARI and/or ICU, obtained from the irritability-by-CU traits-by-Direction-by-Type-by-Emotion repeated measures ANCOVA.

\begin{tabular}{|c|c|c|c|c|c|c|c|}
\hline Region $^{\mathbf{a}}$ & BA & Voxels & $\mathbf{x}$ & $\mathbf{Y}$ & $\mathbf{Z}^{\mathbf{b}}$ & $F$-value & $\eta_{\mathbf{p}}^{2}$ \\
\hline \multicolumn{8}{|c|}{ Irritability-by-CU traits-by-Direction-by-Emotion } \\
\hline $\mathrm{R}$ thalamus/PAG & - & 37 & 11 & -37 & 5 & 34.12 & 0.262 \\
\hline$L$ lingual gyrus & 18 & 33 & -22 & -55 & 2 & 22.47 & 0.190 \\
\hline R fusiform gyrus & 36 & 24 & 38 & -34 & -25 & 27.95 & 0.225 \\
\hline $\operatorname{LACC} C^{\star \star \star}$ & - & 208 & -13 & 41 & 8 & 18.08 & 0.159 \\
\hline$L^{P C C} C^{\star \star \star}$ & 31 & 40 & -10 & -52 & 26 & 16.19 & 0.144 \\
\hline \multicolumn{8}{|l|}{ Irritability-by-Direction-by-Emotion } \\
\hline L ACC & 24 & 26 & -4 & 35 & 5 & 18.36 & 0.161 \\
\hline LPCC & - & 21 & -13 & -46 & 23 & 27.53 & 0.223 \\
\hline R amygdala/parahippocampal gyrus $s^{\star \star \star ~}$ & - & 50 & 20 & -16 & -19 & 26.70 & 0.218 \\
\hline
\end{tabular}

${ }^{a}$ According to the Talairach Daemon Atlas (http://www.nitrc.org/projects/tal-daemon/), ${ }^{b}$ Based on the Tournoux and Talairach standard brain template. All results presented at $p<$ 0.001 (corrected $p<0.05)$ except ${ }^{\star \star *}(p<0.005) \cdot \eta_{p}^{2}=$ partial eta squared.

of Direction, Type, and Emotion and interactions between these variables are reported in Supplementary Table 1.

Irritability-by-CU traits-by-Direction-by-Emotion interaction was significant in three regions: right PAG/thalamus $\left[F_{(1,96)}=\right.$ $\left.34.12 ; p<0.001 ; \eta_{p}^{2}=0.262\right]$, left lingual gyrus [BA 18, $F_{(1,96)}$ $=22.47 ; p<0.001 ; \eta_{p}^{2}=0.190$ ], and right fusiform gyrus [BA $\left.36, F_{(1,96)}=27.95 ; p<0.001 ; \eta_{p}^{2}=0.225\right]$. Across all the three regions, via the PROCESS macro, we determined that a core component of this interaction reflected a differential response to Looming Threatening trials as opposed to Receding Threatening trials. Irritability was significantly positively associated with differential BOLD response to Looming Threatening vs. Receding Threatening trials but at relatively low levels of CU traits (ICU $<20,20,15$; partial $r=0.37,0.27,0.51$; $p=0.013$, $0.068,0.017$ for the three regions, respectively). In contrast, $\mathrm{CU}$ traits were significantly negatively associated with differential BOLD response to Looming Threat vs. Receding Threat trials for higher ARI scores (ARI > 3, 4; partial $r=-0.45,-0.58$; $p=0.028,0.015)$ for right thalamus/PAG and right fusiform gyrus (Figure 2).

A significant irritability-by-Direction-by-Emotion interaction in left ventral anterior cingulate cortex [ACC; BA 24, $F_{(1,96)}$ $\left.=18.36 ; p<0.001 ; \eta_{p}^{2}=0.161\right]$ and left PCC $\left[F_{(1,96)}\right.$ $=27.53 ; p<0.001 ; \eta_{p}^{2}=0.223$ ] was obtained. Within these two regions, irritability was positively correlated with differential BOLD responses to Looming Threats relative to Receding Threats (partial $r=0.21,0.26 ; p=0.039,0.01$; Figure 3) and Looming Neutral stimuli (partial $r=0.29$, $0.28 ; p=0.003,0.004)$. It should be noted that both these regions showed irritability-by-CU traits-by-Direction-by-Emotion interactions albeit at a slightly more liberal initial threshold $(p<$ 0.005; see Supplementary Figure 1). Again, the PROCESS macro revealed that irritability was significantly positively associated with differential BOLD response to Looming Threatening vs. Receding Threatening trials but at relatively low CU traits (ICU $<20$, 22; partial $r=0.39,0.38 ; p=0.007,0.004$ ). In contrast, CU traits were again significantly negatively associated with differential BOLD response to Looming Threatening vs.
Receding Threatening trials particularly for higher ARI scores (ARI $>7,1$; partial $r=-0.76,-0.34 ; p=0.048,0.013$; see Supplementary Figure 1).

\section{Functional ROI Analysis: BOLD Responses to Task Parameters in an Independent Sample of TD Participants}

The ANOVA revealed regions showing main effects of Direction, Type and Emotion. Consistent with previous studies, regions showing increased responses to Looming vs. Receding and Threatening vs. Neutral images included bilateral thalamus, bilateral visual and temporal cortices, bilateral amygdala and vmPFC (though not PCC); see Supplementary Table 2.

\section{Association of CU Traits and Irritability in the Test Sample With Activity Within These Functional ROls}

The ANCOVAs for both the Direction (Looming $>$ Receding) and Emotion (Threatening $>$ Neutral) regions revealed significant irritability-by-CU traits-by-Direction-by-Emotion interactions $\left[\mathrm{F}_{(1,96)}=8.23\right.$ and 5,$43 ; p=0.005$ and $0.022 ; \eta_{p}^{2}=$ 0.08 and 0.05 , respectively). The Process macro revealed that across the regions showing a main effect of Direction, irritability was significantly positively associated with differential BOLD response to Looming Threatening vs. Looming Neutral trials but only at lower levels of CU traits (ICU $<21$ and 20; partial $r=$ 0.33 and $0.31 ; p=0.018$ and $p=0.028$, respectively).

\section{Behavioral Data}

The ANCOVA conducted on the RT data revealed a main effect of Direction $\left[F_{(1,96)}=72.51 ; p<0.001 ; \eta p^{2}=0.430\right.$; participants were faster to respond to Receding than Looming stimuli [386 vs. $419 \mathrm{~ms}])$ and $\mathrm{CU}$ traits $\left[F_{(1,96)}=6.54 ; p=0.012 ; \eta p^{2}=\right.$ 0.064 ; CU traits were positively associated with RTs $(r=0.22$, $p=0.026)]$. In addition, there was a significant irritability-byCU traits interaction $\left[F_{(1,96)}=5.04 ; p=0.027 ; \eta p^{2}=0.050\right]$. The PROCESS macro (49) revealed that the increase in RT as a function of CU traits was greater for individuals with an ARI score $>1$. 


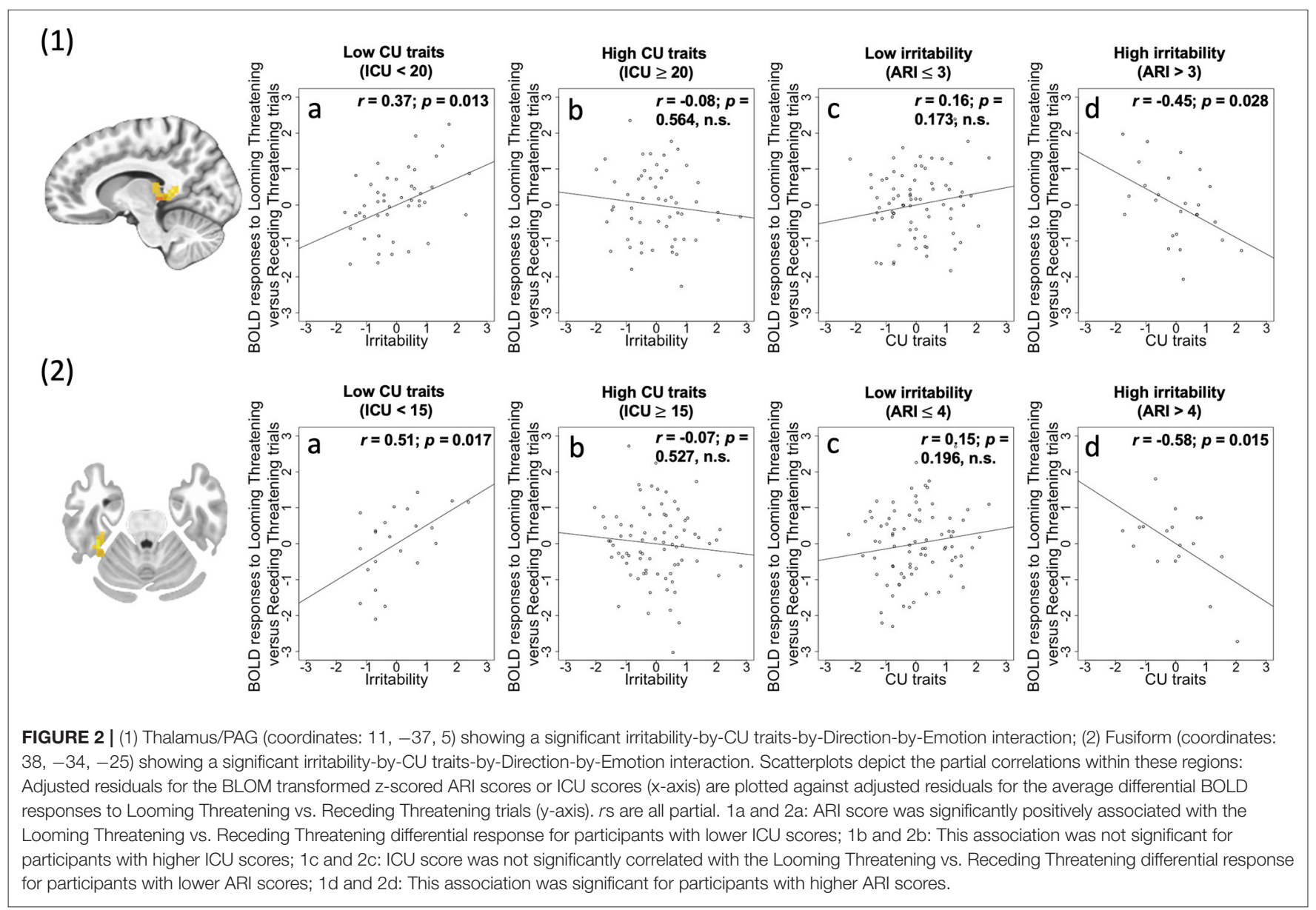

\section{Secondary fMRI Analyses: CD Diagnosis and Potential Confounds CD Diagnosis}

Characteristics of the groups are reported in Table 1. Our Group-based ANOVA revealed: (i) a Group-by-Direction-byType-by-Emotion interaction within left/right cuneus (BA 18 and $\mathrm{BA} 19, F_{(1,103)}=20.68 ; p<0.001 ; \eta p^{2}=0.167$, see Supplementary Table 3). Within this region, the CD participants, compared to the TD participants, showed less of an increase in response to Looming Threatening Animals relative to Receding Threatening Animals $\left[t_{(103)}=2.467, p=0.015\right.$; M[TD] $=0.185 ; \mathrm{M}[\mathrm{CD}]=-0.011]$; and (ii) a Group-by-Type interaction within right $\mathrm{PCC}\left[\mathrm{BA} 30, F_{(1,103)}=19.34 ; p<0.001 ; \eta p^{2}=0.158\right.$, see Supplementary Table 3). Within this region, CD participants showed significantly less of a discrimination between Human and Animal stimuli than TD participants $\left(\mathrm{M}\left[\mathrm{TD}_{\text {Animal }}\right]=0.109\right.$; $\mathrm{M}\left[\mathrm{TD}_{\text {Human }}\right]=0.032 ; \mathrm{M}\left[\mathrm{CD}_{\text {Animal }}\right]=0.040 ; \mathrm{M}\left[\mathrm{CD}_{\text {Human }}\right]$ $=0.049$ ).

\section{Potential Treatment Confounds}

Given the significant associations of ICU and ARI scores with prescription of antipsychotic and/or SSRI medications (see Table 1), our ANCOVA analysis was repeated twice, once excluding participants prescribed SSRIs $(N=10)$ and once excluding participants prescribed antipsychotics $(N=$ 8 ). These analyses largely mirrored the results of our main analysis (for full details, see Supplementary Tables 4, 5). For completion the analysis was conducted a third time excluding participants prescribed stimulants $(N=14)$. Again this analysis largely mirrored the results of our main analysis (see Supplementary Table 6).

\section{The Confound of Potential Suppressor Effects}

To ensure that the effects reported above could not be attributed to suppressor effects, we re-ran our main analysis twice; once with only Rankit-transformed ARI score as a covariate, a second time with only Rankit-transformed ICU score as a covariate. The ARI ANCOVA largely mirrored the regions showing irritability-by-Direction-by-Emotion interactions (see Supplementary Table 7). The ICU ANCOVA showed CU traitsby-Direction-by-Type interactions (see Supplementary Table 8).

\section{DISCUSSION}

In this study, we investigated the extent to which there is an interactive association between level of CU traits and irritability in adolescents with respect to neural systems responsive to threat in a sample representing the range from typically developing to 

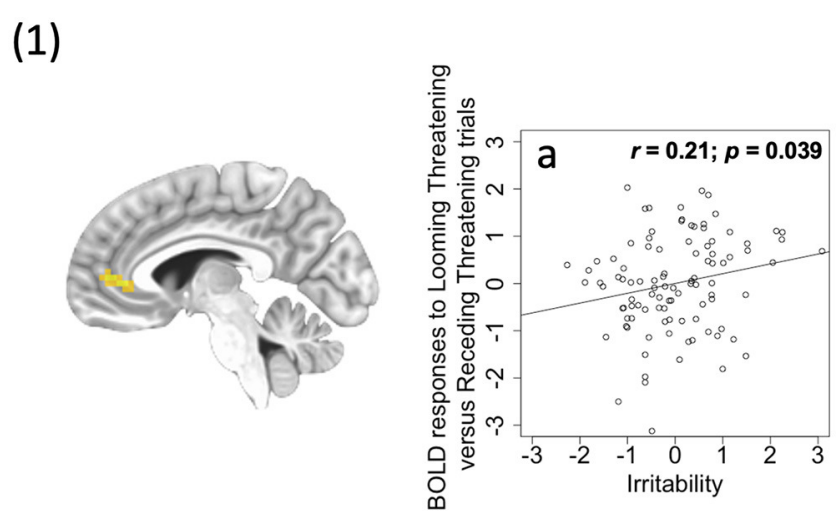

(2)
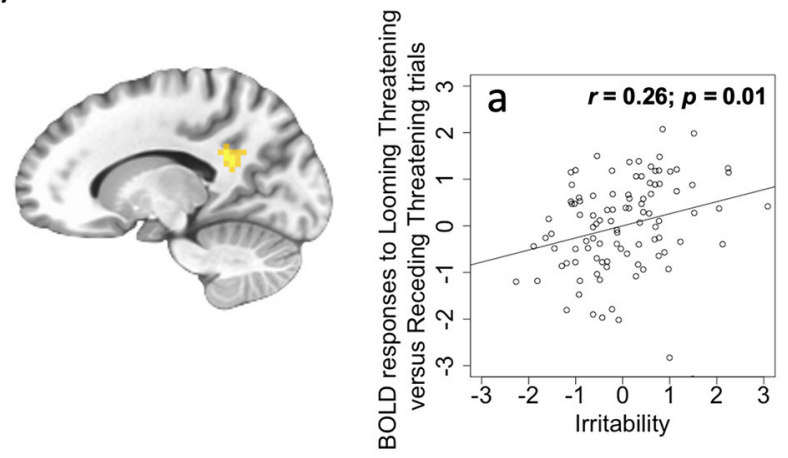

FIGURE 3 | (1) Ventral ACC (coordinates: 26, -4, 35) showing a significant irritability-by-Direction-by-Emotion interaction; (2) PCC (coordinates: 21, -13, -46) showing a significant irritability-by-Direction-by-Emotion interaction. Scatterplots depict the partial correlations within these regions: Adjusted residuals for the BLOM transformed z-scored ARI scores (x-axis) are plotted against adjusted residuals for the average differential BOLD responses to Looming Threatening vs. Receding Threatening trials (y-axis). rs are all partial. 1a and 2a: ARI score was significantly positively associated with the Looming Threatening vs. Receding Threatening differential response.

cases with CD (i.e., a psychiatric condition particularly associated with CU traits and irritability). There were two main findings: (1) Across right thalamus/PAG, left lingual gyrus and right fusiform gyrus, irritability was significantly positively associated with differential BOLD response to Looming Threatening vs. Receding Threatening trials but at relatively low CU traits. In contrast, $\mathrm{CU}$ traits were significantly negatively associated with differential BOLD response to Looming Threatening vs. Receding Threatening trials particularly for individuals with higher ARI scores for right thalamus/PAG and right fusiform gyrus; and (2) Irritability was significantly positively associated with responding within left ventral ACC and left PCC to Looming Threats relative to Receding Threats (though, at slightly more lenient initial thresholds, both regions were showing the same interactions of irritability and $\mathrm{CU}$ traits as right thalamus/PAG, left lingual gyrus and right fusiform gyrus).

We predicted that irritability would be related to heightened threat responsiveness within regions involved in responding to threat (the amygdala, PAG and connected cortical regions).
Previous work has reported heightened responsiveness within these regions to emotional stimuli in participants with elevated irritability (23-27). In the current study, irritability (at least in those with lower CU traits) was associated with increased responses to approaching threats (relative to comparison conditions) within the amygdala and PAG, fusiform and lingual gyrus and ventral ACC and PCC (albeit with respect to the amygdala at a slightly more liberal threshold). In short, irritability was associated with increased responses within regions included in the acute threat response [amygdala and PAG; $(4,50)$ ], regions representing visual stimuli that are recipients of priming from the amygdala to emotional stimuli [lingual and fusiform cortices; $(26,27)]$ and regions representing the value of emotional stimuli [and perhaps organizing regulatory responding; vACC and PCC; (51-54)]. These findings are consistent with a view that a core component of irritability is a dysregulated acute threat response such that the individual is more likely to express reactive aggression to a provocation than freezing or avoidance $(5,50,55)$.

However, it should be noted that for these regions, the association between irritability and threat responsiveness was moderated by level of CU traits (though for some at slightly more liberal thresholds). Specifically, the positive relationship between ARI score and threat responsiveness was more marked for those with lower CU traits. In most respects, this was anticipated. ARI score and CU traits have been relatively consistently related to inverse forms of psychopathology; increased vs. decreased threat responsiveness, respectively $(4,15)$. Yet, both are associated with similar symptoms - specifically, anger and reactive aggression $(4,15,56)$. It is argued that irritability and reactive aggression can result from increased threat responsiveness; the individual responds with rage rather than freeze or flight to provocation $(5,50,55)$. But it is also argued that reactive aggression and anger can result from dysfunctional modulation of threat response circuitry and impaired decision-making regarding the value of future response options; the individual fails to represent the "badness" of the action and so is more likely to commit the non-optimal act (4). We assume that for participants showing irritability, but with low CU traits, their level of irritability reflected the existent hyper-threat responsive psychopathology. However, for many of the participants with higher levels of irritability, the pathology underling their behavior might reflect either hyper- or hypo- threat responsiveness. Indeed, within participants with high ARI scores, the negative association between $\mathrm{CU}$ traits and threat responsiveness was most marked. Amongst these individuals, we would argue, the more CU traits the individual showed, the more their reactive aggression and anger was driven by the pathophysiology underpinning CU traits; i.e., dysfunctional representation of the "badness" of anger displays (i.e., the "victims" distress at the anger display and/or the negative consequences following displays of anger for the displayer).

It is worth briefly considering development. Within the sample itself, age plays a relatively minor role (the range is rather narrow 14-18 years; see Supplementary Table 9). Moreover, our main finding, that increased threat responsiveness was associated with irritability unless participants were showing clinically significant levels of CU traits, is unlikely to be highly 
age-dependent. There are no established theoretical reasons for why this interaction might manifest differently across development. However, heightened threat responsiveness could reflect a failure to mature appropriate emotion regulation mechanisms. Emotion regulation capacities develop substantially across adolescence (57-59). The heightened threat responsiveness might reflect a relative failure in emotion regulation development that can put the individual at increased risk for irritability.

The results for the Group-based ANOVA are worth a brief note. Previous work with aggressive adolescents has mostly focused on distress cues, as opposed to threat responsiveness, in youth with disruptive behavior disorders (DBDs) (60-62). However, there have been studies focusing on responses to threat stimuli in youth with DBDs $(16,63-66)$. Most of these studies have reported reduced threat responsiveness in adolescents with $\mathrm{CD} /$ oppositional defiant disorder [though see $(63,66)]$. Our finding of reduced responsiveness in the adolescents with $\mathrm{CD}$ relative to comparison adolescents to Looming, Threatening Animals relative to Receding, Threatening Animals within left/right cuneus is consistent with this literature. Importantly, though, this hints that the pathophysiology underpinning the slight majority of cases in this sample was the reduced threat responsiveness putatively underpinning $\mathrm{CU}$ traits (as the group of adolescents with CD showed some indication of reduced threat responsiveness). Irritability is seen as a highly cross-diagnostic trait and reported to be increased not only in those showing conduct problems but frequently in cases with mood and anxiety conditions $(11,19,67)$. A unique feature of CD is the increased risk for instrumental, goal-directed aggression, something not typically associated with irritability but highly associated with CU traits (4). Of course, as our data reflect, a significant proportion of participants with $\mathrm{CD}$ are instead underpinned by the heightened threat responsiveness, the pathophysiology purportedly associated with irritability. As such, it is possible that locations sampling CD populations whose predominant feature is irritability rather than $\mathrm{CU}$ traits might report rather different results [cf. (63)].

The current results have clinical implications. Externalizing behaviors are a leading cause of child and adolescent referrals to mental health clinicians (68). Both CU traits and irritability are commonly associated with externalizing behavior $(10,69)$. The current data suggest a treatment target for irritability if CU traits are low-heightened threat responsiveness. Treatments reducing threat responsiveness might be useful for patients with elevated irritability but not co-presenting CU traits [e.g., (70, 71)]. But, importantly, the current data suggest the situation is more complex if the patient also presents with $\mathrm{CU}$ traits at a clinically concerning level. Indeed, the current data suggest that amongst patients with higher irritability, the greater the level of their CU traits, the less hyper-threat responsiveness is a problem. Indeed, amongst these patients, increasing, rather than decreasing emotionality, might be a treatment target [cf. (33)] - though it is unclear currently how this might be achieved.

Four caveats should be considered with respect to the current results. First, there were significant associations between ICU and ARI scores and prescriptions of antipsychotic and/or SSRI medications (see Table 1). As such, the current results might reflect influence of these prescriptions rather than irritability and/or CU traits. Ameliorating this concern is the fact that the secondary analyses that we conducted with participants prescribed either antipsychotic or SSRI medications excluded did not significantly change our results (see Supplementary Tables 4, 5). These data suggest prescribed medications did not significantly confound the current results. Second, one surprising feature in the results was that while $\mathrm{CU}$ traits were significantly negatively related to threat responsiveness in participants with higher irritability, they were not in participants with lower irritability. We would have expected that $\mathrm{CU}$ traits would have been negatively related to threat responsiveness across the range of irritability scores. Future work will be necessary to determine the robustness of these data and their interpretation. Third, diagnoses followed clinical practice that included an interview by a board-certified psyhchiatrist rather than the implementation of a structured or semi-structured diagnostic interview. While this could raise concern regarding the $\mathrm{CD}$ diagnoses, it is important to note: (i) that these diagnoses were supported by the SDQ-CP scores; and (ii) that the main goal of this work was to investigate neural signatures related to CU traits and irritability across the sample irrespective of diagnostic status/comorbid conditions. Fourth, there were associations between AUD and CUD symptom severity and both $\mathrm{CU}$ traits and irritability. However, it is important to note that the strengths of these associations did not differ (ICU vs. ARI). As such, AUD and CUD symptom severity is less likely to underpin the dissociable CU trait and irritability effects reported here.

In conclusion, irritability was associated with increasing responsiveness in regions that manifest three different functions in this study: (1) thalamus/PAG and amygdala generating response to threat, (2) lingual gyrus and fusiform gyrus representing threat visually, and (3) ACC and PCC maintaining emotional valence and emotional reaction. For most of these regions, this association was moderated by level of $\mathrm{CU}$ traits and was most marked for participants with low CU traits. For participants showing higher levels of irritability, CU traits were inversely related to emotional responsiveness for many of these regions. These data indicate that high levels of irritability might be manifested as a consequence of heightened threat responsiveness but also due to the pathophysiology associated with CU traits.

\section{DATA AVAILABILITY STATEMENT}

The original contributions presented in the study are included in the article/Supplementary Material, further inquiries can be directed to the corresponding author/s.

\section{ETHICS STATEMENT}

The studies involving human participants were reviewed and approved by the Boys Town National Research Hospital Institutional Review Board. Written informed consent to 
participate in this study was provided by the participants' legal guardian/next of kin.

\section{AUTHOR CONTRIBUTIONS}

All authors have contributed in a scientifically substantial way to this manuscript.

\section{FUNDING}

This research was in part supported by the National Institute of Mental Health under award number K22MH109558 (JB-L). The funders had no role in the design and conduct of the study; collection, management, analysis, and interpretation of the data; preparation, review, or approval

\section{REFERENCES}

1. APA (2013). Diagnostic and Statistical Manual of Mental Disorders (5th ed.). Arlington, VA: American Psychiatric Publishing.

2. Fairchild G, Hawes DJ, Frick PJ, Copeland WE, Odgers CL, Franke B, et al. Conduct disorder. Nat Rev Dis Primers. (2019) 5:43. doi: 10.1038/s41572-019-0095-y

3. Loeber R, Burke JD, Pardini DA. Development and etiology of disruptive and delinquent behavior. Annu Rev Clin Psychol. (2009) 5:291-310. doi: 10.1146/annurev.clinpsy.032408.153631

4. Blair RJR. Traits of empathy and anger: Implications for psychopathy and other disorders associated with aggression. Philos Trans R Soc Lond B Biol Sci. (2018) 373:20170155. doi: 10.1098/rstb.2017.0155

5. Blair RJR, Veroude K, Buitelaar JK. Neuro-cognitive system dysfunction and symptom sets: a review of fMRI studies in youth with conduct problems. Neurosci Biobehav Rev. (2018) 91:69-90. doi: 10.1016/j.neubiorev.2016.10.022

6. Kragel PA, LaBar KS. Decoding the nature of emotion in the brain. Trends Cogn Sci. (2016) 20:444-55. doi: 10.1016/j.tics.2016.03.011

7. LeDoux JE. The amygdala. Curr Biol. (2007) 17:R86874. doi: 10.1016/j.cub.2007.08.005

8. Mobbs D, Marchant JL, Hassabis D, Seymour B, Tan G, Gray M, et al. From threat to fear: the neural organization of defensive fear systems in humans. $J$ Neurosci. (2009) 29:12236-43. doi: 10.1523/JNEUROSCI.2378-09.2009

9. Panksepp J. Affective Neuroscience: The Foundations of Human and Animal Emotions. Oxford: Oxford University Press (1998).

10. Frick PJ, Ray JV, Thornton LC, Kahn RE. Annual research review: a developmental psychopathology approach to understanding callousunemotional traits in children and adolescents with serious conduct problems. J Child Psychol Psychiatry. (2014) 55:532-48. doi: 10.1111/jcpp.12152

11. Leibenluft E. Pediatric irritability: a systems neuroscience approach. Trends Cogn Sci. (2017) 21:277-89. doi: 10.1016/j.tics.2017.02.002

12. Camacho MC, Karim HT, Perlman SB. Neural architecture supporting active emotion processing in children: a multivariate approach. Neuroimage. (2019) 188:171-80. doi: 10.1016/j.neuroimage.2018.12.013

13. Fishburn FA, Hlutkowsky CO, Bemis LM, Huppert TJ, Wakschlag LS, Perlman SB. Irritability uniquely predicts prefrontal cortex activation during preschool inhibitory control among all temperament domains: a LASSO approach. Neuroimage. (2019) 184:68-77. doi: 10.1016/j.neuroimage.2018.09.023

14. Stringaris A, Goodman R, Ferdinando S, Razdan V, Muhrer E, Leibenluft E, et al. The affective reactivity index: a concise irritability scale for clinical and research settings. J Child Psychol Psychiatry. (2012) 53:110917. doi: 10.1111/j.1469-7610.2012.02561.x

15. Wakschlag LS, Perlman SB, Blair RJ, Leibenluft E, Briggs-Gowan MJ, Pine DS. The neurodevelopmental basis of early childhood disruptive behavior: Irritable and callous phenotypes as exemplars. Am J Psychiatry. (2018) 175:114-30. doi: 10.1176/appi.ajp.2017.17010045 of the manuscript; and decision to submit the manuscript for publication.

\section{ACKNOWLEDGMENTS}

We would like to thank Ron Copsey, Kim VanHorn, Michael Wright, Mark Timm, and Rhonda Tuel for their contributions to data collection. We would like to thank all participants and their families for their participation.

\section{SUPPLEMENTARY MATERIAL}

The Supplementary Material for this article can be found online at: https://www.frontiersin.org/articles/10.3389/fpsyt. 2021.617052/full\#supplementary-material

16. Hwang S, Nolan ZT, White SF, Williams WC, Sinclair S, Blair RJ. Dual neurocircuitry dysfunctions in disruptive behavior disorders: emotional responding and response inhibition. Psychol Med. (2016) 46:1485-96. doi: 10.1017/S0033291716000118

17. Sebastian CL, McCrory EJ, Dadds MR, Cecil CA, Lockwood PL, Hyde ZH, et al. Neural responses to fearful eyes in children with conduct problems and varying levels of callous-unemotional traits. Psychol Med. (2014) 44:99109. doi: 10.1017/S0033291713000482

18. White SF, Williams WC, Brislin SJ, Sinclair S, Blair KS, Fowler KA, et al. Reduced activity within the dorsal endogenous orienting of attention network to fearful expressions in youth with disruptive behavior disorders and psychopathic traits. Dev Psychopathol. (2012) 24:110516. doi: 10.1017/S0954579412000569

19. Brotman MA, Kircanski K, Stringaris A, Pine DS, Leibenluft E. Irritability in youths: a translational model. Am J Psychiatry. (2017) 174:52032. doi: 10.1176/appi.ajp.2016.16070839

20. Blair RJR, Bajaj S, Sherer N, Bashford-Largo J, Zhang R, Aloi J, et al. (2021). Alcohol use disorder and cannabis use disorder symptomatology in adolescents and aggression: associations with recruitment of neural regions implicated in retaliation. Biol Psychiatry Cogn Neurosci Neuroimaging, 6:536544. doi: 10.1016/j.bpsc.2020.11.016

21. Barker ED, Salekin RT. Irritable oppositional defiance and callous unemotional traits: is the association partially explained by peer victimization? J Child Psychol Psychiatry. (2012) 53:116775. doi: 10.1111/j.1469-7610.2012.02579.x

22. Stringaris A, Zavos H, Leibenluft E, Maughan B, Eley TC. Adolescent irritability: phenotypic associations and genetic links with depressed mood. Am J Psychiatry. (2012) 169:47-54. doi: 10.1176/appi.ajp.2011.10101549

23. Crum KI, Hwang S, Blair KS, Aloi JM, Meffert H, White SF, et al. Interaction of irritability and anxiety on emotional responding and emotion regulation: a functional MRI study. Psychol Med. (2020) 25:111. doi: 10.1017/S0033291720001397

24. Kircanski K, White LK, Tseng WL, Wiggins JL, Frank HR, Sequeira S, et al. A latent variable approach to differentiating neural mechanisms of irritability and anxiety in youth. JAMA Psychiatry. (2018) 75:6319. doi: 10.1001/jamapsychiatry.2018.0468

25. McCloskey MS, Phan KL, Angstadt M, Fettich KC, Keedy S, Coccaro EF. Amygdala hyperactivation to angry faces in intermittent explosive disorder. J Psychiatr Res. (2016) 79:34-41. doi: 10.1016/j.jpsychires.2016.04.006

26. Stoddard J, Tseng WL, Kim P, Chen G, Yi J, Donahue L, et al. Association of irritability and anxiety with the neural mechanisms of implicit face emotion processing in youths with psychopathology. JAMA Psychiatry. (2017) 74:95103. doi: 10.1001/jamapsychiatry.2016.3282

27. Wiggins JL, Brotman MA, Adleman NE, Kim P, Oakes AH, Reynolds RC, et al. Neural correlates of irritability in disruptive mood dysregulation and bipolar disorders. Am J Psychiatry. (2016) 173:722-30. doi: 10.1176/appi.ajp.2015.15060833 
28. Cuthbert BN, Insel TR. Toward the future of psychiatric diagnosis: the seven pillars of RDoC. BMC Med. (2013) 11:126. doi: 10.1186/1741-7015-11-126

29. Insel TR. The NIMH research domain criteria (RDoC) project: precision medicine for psychiatry. Am J Psychiatry. (2014) 171:395-7. doi: 10.1176/appi.ajp.2014.14020138

30. Coker-Appiah DS, White SF, Clanton R, Yang J, Martin A, Blair RJ. Looming animate and inanimate threats: the response of the amygdala and periaqueductal gray. Soc Neurosci. (2013) 8:621-30. doi: 10.1080/17470919.2013.839480

31. Blair KS, Bashford-Largo J, Shah N, Lukoff J, Elowsky J, Vogel S, et al. Sexual abuse in adolescents is associated with atypically increased responsiveness within regions implicated in self-referential and emotional processing to approaching animate threats [Original Research]. Front Psychiatry. (2020) 11:345 doi: 10.3389/fpsyt.2020.00345

32. Blair KS, Bashford-Largo J, Shah N, Lukoff J, Thompton LC, Leiker EK, et al. Threat responsiveness as a function of cannabis and alcohol use disorder severity. J Child Adolesc Psychopharmacol. (2019) 29:52634. doi: 10.1089/cap.2019.0004

33. Blair RJ, Zhang R, Bashford-Largo J, Bajaj S, Mathur A, Ringle J, et al. Reduced neural responsiveness to looming stimuli is associated with increased aggression. Soc Cogn Affect Neurosci. (2021) 16:109199. doi: $10.1093 / \mathrm{scan} / \mathrm{nsab} 058$

34. White SF, Thornton LC, Leshin J, Clanton R, Sinclair S, Coker-Appiah $\mathrm{D}$, et al. Looming threats and animacy: reduced responsiveness in youth with disrupted behavior disorders. J Abnorm Child Psychol. (2018) 46:74154. doi: 10.1007/s10802-017-0335-0

35. Frick PJ. Inventory of Callous-Unemotional Traits. University of New Orleans (2004).

36. Essau CA, Sasagawa S, Frick PJ. Callous-unemotional traits in a community sample of adolescents. Assessment. (2006) 13:459-69. doi: 10.1177/1073191106287354

37. Kimonis ER, Frick PJ, Skeem JL, Marsee MA, Cruise K, Munoz LC. Assessing callous-unemotional traits in adolescent offenders: validation of the inventory of callous-unemotional traits. Int J Law Psychiatry. (2008) 31:241-52. doi: 10.1016/j.ijlp.2008.04.002

38. Goodman R. The strengths and difficulties questionnaire: a research note. J Child Psychol Psychiatry. (1997) 38:5816. doi: 10.1111/j.1469-7610.1997.tb01545.x

39. Raine A, Dodge K, Loeber R, Gatzke-Kopp L, Lynam D, Reynolds C, et al. The reactive-proactive aggression questionnaire: differential correlates of reactive and proactive aggression in adolescent boys. Aggress Behav. (2006) 32:15971. doi: $10.1002 / \mathrm{ab} .20115$

40. Conners CK. Clinical use of rating scales in diagnosis and treatment of attention-deficit/hyperactivity disorder. Pediatr Clin North Am. (1999) 46:857-70, vi. doi: 10.1016/S0031-3955(05)70159-0

41. Saunders JB, Aasland OG, Babor TF, de la Fuente JR, Grant M. Development of the alcohol use disorders identification test (AUDIT): WHO collaborative project on early detection of persons with harmful alcohol consumption-II. Addiction. (1993) 88:791-804. doi: 10.1111/j.1360-0443.1993. tb02093.x

42. Adamson SJ, Kay-Lambkin FJ, Baker AL, Lewin TJ, Thornton L, Kelly BJ, et al. An improved brief measure of cannabis misuse: the Cannabis Use Disorders Identification Test-Revised (CUDIT-R). Drug Alcohol Depend. (2010) 110:137-43. doi: 10.1016/j.drugalcdep.2010.02.017

43. Cox RW. AFNI: Software for analysis and visualization of functional magnetic resonance neuroimages. Comput Biomed Res. (1996) 29:16273. doi: 10.1006/cbmr.1996.0014

44. Talairach J, Tournoux P. Co-Planar Stereotaxic Atlas of the Human Brain. New York, NY: Thieme (1988).

45. Bliss CI, Greenwood ML, White ES. A Rankit analysis of paired comparisons for measuring the effect of sprays on flavor. Biometrics. (1956) 12:381403. doi: $10.2307 / 3001679$

46. Steiger JH. Tests for comparing elements of a correlation matrix. Psychol Bull. (1980) 87:245-51. doi: 10.1037/0033-2909.87.2.245

47. Preacher KJ, Hayes AF. SPSS and SAS procedures for estimating indirect effects in simple mediation models. Behav Res Methods, Instruments, Comput. (2004) 36:717-31. doi: 10.3758/BF03206553
48. Kowalski CJ, Schneiderman ED, Willis SM. ANCOVA for nonparallel slopes: the Johnson-Neyman technique. Int J Biomed Comput. (1994) 37:27386. doi: 10.1016/0020-7101(94)90125-2

49. Hayes A. Introduction to Mediation, Moderation, and Conditional Process Analysis: A Regression-Based Approach. New York, NY: Guilford Press (2013).

50. Blair RJR. Considering anger from a cognitive neuroscience perspective. Wiley Interdiscip Rev Cogn Sci. (2012) 3:65-74. doi: 10.1002/wcs.154

51. Wager TD, van Ast VA, Hughes BL, Davidson ML, Lindquist MA, Ochsner $\mathrm{KN}$. Brain mediators of cardiovascular responses to social threat, part II: Prefrontal-subcortical pathways and relationship with anxiety. Neuroimage. (2009) 47:836-51. doi: 10.1016/j.neuroimage.2009.05.044

52. Waugh CE, Lemus MG, Gotlib IH. The role of the medial frontal cortex in the maintenance of emotional states. Soc Cogn Affect Neurosci. (2014) 9:2001-9. doi: 10.1093/scan/nsu011

53. Waugh CE, Schirillo JA. Timing: a missing key ingredient in typical fMRI studies of emotion. Behav Brain Sci. (2012) 35:170-1. doi: 10.1017/S0140525X11001646

54. Waugh CE, Zarolia P, Mauss IB, Lumian DS, Ford BQ, Davis TS, et al. Emotion regulation changes the duration of the BOLD response to emotional stimuli. Soc Cogn Affect Neurosci. (2016) 11:1550-9. doi: 10.1093/scan/nsw067

55. Leibenluft E. Irritability in children: what we know and what we need to learn. World Psychiatry. (2017) 16:100-1. doi: 10.1002/wps.20397

56. Pardini DA, Frick PJ. Multiple developmental pathways to conduct disorder: current conceptualizations and clinical implications. J Can Acad Child Adolesc Psychiatry. (2013) 22:20-25. doi: 10.1080/15374416.2012.664815

57. Gullone E, Hughes EK, King NJ, Tonge B. The normative development of emotion regulation strategy use in children and adolescents: a 2-year follow-up study. J Child Psychol Psychiatry. (2010) 51:567-74. doi: 10.1111/j.1469-7610.2009.02183.x

58. Young KS, Sandman CF, Craske MG. Positive and negative emotion regulation in adolescence: links to anxiety and depression. Brain Sci. (2019) 9:76. doi: 10.3390/brainsci9040076

59. Zimmermann P, Iwanski A. Emotion regulation from early adolescence to emerging adulthood and middle adulthood: age differences, gender differences, and emotion-specific developmental variations. Int J Behav Dev. (2014) 38:182-94. doi: 10.1177/0165025413515405

60. Jones AP, Laurens KR, Herba CM, Barker GJ, Viding E. Amygdala hypoactivity to fearful faces in boys with conduct problems and callous-unemotional traits. Am J Psychiatry. (2009) 166:95-102. doi: 10.1176/appi.ajp.2008.07071050

61. Marsh AA, Finger EC, Mitchell DG, Reid ME, Sims C, Kosson DS, et al. Reduced amygdala response to fearful expressions in children and adolescents with callous-unemotional traits and disruptive behavior disorders. Am J Psychiatry. (2008) 165:712-20. doi: 10.1176/appi.ajp.2007.07071145

62. Passamonti L, Fairchild G, Goodyer IM, Hurford G, Hagan CC, Rowe JB, et al. Neural abnormalities in early-onset and adolescence-onset conduct disorder. Arch Gen Psychiatry. (2010) 67:729-38. doi: 10.1001/archgenpsychiatry.2010.75

63. Fehlbaum LV, Raschle NM, Menks WM, Pratzlich M, Flemming E, Wyss L, et al. Altered neuronal responses during an affective stroop task in adolescents with conduct disorder. Front Psychol. (2018) 9:1961. doi: 10.3389/fpsyg.2018.01961

64. Herpertz SC, Huebner T, Marx I, Vloet TD, Fink GR, Stoeker T, et al. Emotional processing in male adolescents with childhoodonset conduct disorder. J Child Psychol Psychiatry. (2008) 47:781-91. doi: 10.1111/j.1469-7610.2008.01905.x

65. Stadler C, Sterzer P, Schmeck K, Krebs A, Kleinschmidt A, Poustka F. Reduced anterior cingulate activation in aggressive children and adolescents during affective stimulation: association with temperament traits. J Psychiatr Res. (2007) 41:410-7. doi: 10.1016/j.jpsychires.2006.01.006

66. Sterzer P, Stadler C, Krebs A, Kleinschmidt A, Poustka F. Abnormal neural responses to emotional visual stimuli in adolescents with conduct disorder. Biol Psychiatry. (2005) 57:7-15. doi: 10.1016/j.biopsych.2004. 10.008

67. Vidal-Ribas P, Brotman MA, Valdivieso I, Leibenluft E, Stringaris A. The status of irritability in psychiatry: a conceptual and quantitative review. J Am Acad Child Adolesc Psychiatry. (2016) 55:556-70. doi: 10.1016/j.jaac.2016.04.014 
68. Coghill D. Editorial: do clinical services need to take conduct disorder more seriously? J Child Psychol Psychiatry. (2013) 54:921-3. doi: 10.1111/JCPP.12135

69. Sukhodolsky DG, Smith SD, McCauley SA, Ibrahim K, Piasecka JB. Behavioral interventions for anger, irritability, and aggression in children and adolescents. $J$ Child Adolesc Psychopharmacol. (2016) 26:58-64. doi: 10.1089/cap.2015. 0120

70. Hwang S, Chung U, Chang Y, Kim E, Suk JW, Meffert H, et al. Neural responses to fluoxetine in youths with disruptive behavior and trauma exposure: a pilot study. $J$ Child Adolesc Psychopharmacol. (2021) 31:562-71. doi: 10.1089/cap.2020. 0174

71. Towbin K, Vidal-Ribas P, Brotman MA, Pickles A, Miller KV, Kaiser A, et al. A double-blind randomized placebo-controlled trial of citalopram adjunctive to stimulant medication in youth with chronic severe irritability. J Am Acad Child Adolesc Psychiatry. (2020) 59:350-61. doi: 10.1016/j.jaac.2019. 05.015
Conflict of Interest: The authors declare that the research was conducted in the absence of any commercial or financial relationships that could be construed as a potential conflict of interest.

Publisher's Note: All claims expressed in this article are solely those of the authors and do not necessarily represent those of their affiliated organizations, or those of the publisher, the editors and the reviewers. Any product that may be evaluated in this article, or claim that may be made by its manufacturer, is not guaranteed or endorsed by the publisher.

Copyright (c) 2021 Zhang, Bashford-Largo, Lukoff, Elowsky, Carollo, Schwartz, Dobbertin, Bajaj, Blair, Leibenluft and Blair. This is an open-access article distributed under the terms of the Creative Commons Attribution License (CC BY). The use, distribution or reproduction in other forums is permitted, provided the original author(s) and the copyright owner(s) are credited and that the original publication in this journal is cited, in accordance with accepted academic practice. No use, distribution or reproduction is permitted which does not comply with these terms. 